SPIN-2004/05

ITP-UU-04/11

\title{
Emergence of a 4D World from Causal Quantum Gravity
}

\author{
J. Ambjørn ${ }^{a, c}$ J. Jurkiewicz ${ }^{b}$, and R. Loll ${ }^{c}$ \\ ${ }^{a}$ The Niels Bohr Institute, Copenhagen University \\ Blegdamsvej 17, DK-2100 Copenhagen Ø, Denmark. \\ email: ambjorn@nbi.dk \\ ${ }^{b}$ Institute of Physics, Jagellonian University, \\ Reymonta 4, PL 30-059 Krakow, Poland. \\ email: jurkiewicz@th.if.uj.edu.pl \\ ${ }^{c}$ Institute for Theoretical Physics, Utrecht University, \\ Leuvenlaan 4, NL-3584 CE Utrecht, The Netherlands. \\ email: j.ambjorn@phys.uu.nl, r.loll@phys.uu.nl
}

\begin{abstract}
Causal Dynamical Triangulations in four dimensions provide a backgroundindependent definition of the sum over geometries in nonperturbative quantum gravity, with a positive cosmological constant. We present evidence that a macroscopic four-dimensional world emerges from this theory dynamically.
\end{abstract}




\section{Introduction}

String theory has highlighted the fact that the notion of "dimension" in a quantum theory of all fundamental interactions has a very different status from that in any classical field theory or any perturbative quantum field theory on a fixed background, where the dimension of spacetime remains fixed throughout. This may be seen as a particular case of the more general truth, not always appreciated, that in any nonperturbative theory of quantum gravity dimension will become a dynamical quantity, along with other aspects of geometry. (By dimension we mean an effective dimension observed at macroscopic scales.)

This Letter deals with an approach to quantum gravity in which the dynamical property of spacetime dimension is particularly transparent. ${ }^{1}$ The approach is that of "nonperturbative quantum gravity from Lorentzian dynamical triangulations" or, for short, "Causal Dynamical Triangulations". In it, the sum over all spacetime geometries takes the form of a sum over four-geometries constructed from discrete building blocks. ${ }^{2}$ Each collection of building blocks, glued together according to a set of rules, represents a geometry contributing to the path integral. To extract its physical geometric properties one has to perform the path integral, renormalize, and evaluate the expectation values of appropriate observables, including the effective (or Hausdorff) dimension $d_{h}$.

Note that the dynamical nature of "dimensionality" implies that the Hausdorff dimension of the quantum geometry is not a priori determined by the dimensionality at the cut-off scale $a$, which is simply the fixed dimensionality $d$ of the building blocks of the regularized version of the theory. An example in point are the attempts to define theories of quantum geometry via "Euclidean Dynamical Triangulations", much-studied during the 1980s and '90s. In these models, if the dimension $d$ is larger than 2 , and if all geometries contribute to the path integral with equal weight, a geometry with no linear extension and $d_{h}=\infty$ is created with probability one. If instead - as is natural for a gravityinspired theory - the Boltzmann weight of each geometry is taken to be the exponential of (minus) the Euclidean Einstein-Hilbert action, one finds for small values of the bare gravitational coupling constant a first-order phase transition to a phase of the opposite extreme, namely, one in which the quantum geometry satisfies $d_{h}=2$. This is indicative of a different type of degeneracy, where typical

\footnotetext{
${ }^{1}$ An interesting attempt to dynamically derive a four-dimensional spacetime in string theory has been made in the nonperturbative M(atrix) formulation of type IIB strings [1, 2, 3], thus far with no definite conclusion on the dimensionality [4, [5, 6, 7, 8, 9, 10,

${ }^{2}$ We would like to emphasize that the presence of discrete simplicial building blocks does not introduce any fundamental discreteness of spacetime in the theory. The simplices are the analogues in dynamical gravity of the fixed hypercubic lattice cells used to define QCD nonperturbatively. The geodesic length parameter $a$ characterizing their edge lengths serves merely as a short-distance cut-off which is taken to zero in the continuum limit.
} 
(i.e. probability one) configurations are so-called branched polymers or trees (see [11, 12, 13, 14, 15, 16, 17, for details of the phase structure and geometric properties of the four-dimensional Euclidean theory). Unfortunately, this geometric degeneracy is also reflected in the expectation values of other geometric quantities, and makes Euclidean Dynamical Triangulations an unlikely candidate for a theory of four-dimensional quantum gravity.

The requirement that a background-independent quantum gravity theory possess the correct semi-classical limit, given by a macroscopically four-dimensional spacetime with microscopic quantum fluctuations, is highly non-trivial. The apparent failure of higher-dimensional Euclidean nonperturbative quantum gravity to do so (not limited to this particular approach [18]), together with a desire to formulate a quantum gravity with the correct Lorentzian signature and causal properties [19] were our main reasons for suggesting a radically different approach to the sampling of geometries in the path integral [20, 21, 22, 23]. A further motivation was to construct a path integral more closely related to canonical formulations of quantum gravity. Starting from Lorentzian simplicial spacetimes with $d=4$ we insist that only causally well-behaved geometries appear in the (regularized) path integral, as described in detail in [23. A further crucial property of our explicit construction is that each configuration allows for a rotation to Euclidean signature, a necessary prerequisite for discussing the convergence properties of the sum over geometries, as well as for using Monte Carlo techniques.

In what follows we will report on the outcome of the first ever Monte Carlo simulations of four-dimensional causal dynamical triangulations. It differs radically from what was found in previous simulations of four-dimensional Euclidean dynamical triangulations. We will present strong evidence that the Lorentzian framework produces a quantum geometry which is both extended and effectively four-dimensional. This is to our knowledge the first example of a theory of quantum gravity that generates a quantum spacetime with such properties dynamically.

\section{The emergent macroscopic $4 \mathrm{~d}$ geometries}

All causal simplicial spacetimes contributing to the path integral are foliated by an integer-valued "proper time" $t$, and each geometry can be obtained by gluing together four-simplices in a way that respects this foliation. Each four-simplex has timelike links of length-squared $a_{t}^{2}$ and spacelike links of length-squared $a_{s}^{2}$, with all of the latter being located in spatial slices of constant integer- $t$. These slices consist of purely spacelike tetrahedra, forming a three-dimensional piecewise flat manifold, whose topology we choose to be that of a three-sphere $S^{3}$.

Each spatial tetrahedron at time $t$ is shared by two four-simplices (said to be of type $(1,4)$ and $(4,1)$ ) whose fifth vertex lies in the neighbouring slice of time 
$t-1$ and $t+1$ respectively. In addition we need four-simplices of type $(2,3)$ and $(3,2)$ which share one link and one triangle with two adjacent spatial slices [23]. Let us assume the link lengths are related by

$$
a_{t}^{2}=-\alpha a_{s}^{2}
$$

All choices $\alpha>0$ correspond to Lorentzian and all choices $\alpha<-7 / 12$ to Euclidean signature, and a Euclideanization of geometry amounts to a suitable analytic continuation in $\alpha$. Setting $\alpha=-1$ leads to a particularly simple expression for the (Euclidean) Einstein-Hilbert action of a given triangulation $T$ (since all four-simplices are identical geometrically), namely,

$$
S(T)=-k_{0} N_{0}(T)+k_{4} N_{4}(T),
$$

with $N_{i}(T)$ denoting the number of $i$-dimensional simplices in $T$. In (2), $k_{0}$ is proportional to the inverse (bare) gravitational coupling constant, $k_{0} \sim 1 / G$, while $k_{4}$ is a linear combination of the cosmological and inverse gravitational coupling constants. The action (2) has been calculated from Regge's prescription for piecewise linear geometries. If we take $\alpha \neq-1$ the Euclidean four-simplices of type $(1,4)$ and type $(2,3)$ will be different and thus appear with different weights in the Einstein-Hilbert action [23]. For our present purposes it is convenient to use the equivalent parameterization

$$
S(T)=-k_{0} N_{0}(T)+k_{4} N_{4}(T)+\Delta\left(2 N_{14}(T)+N_{23}(T)\right),
$$

where $N_{14}(T)$ and $N_{23}(T)$ denote the combined numbers in $T$ of four-simplices of types $(1,4)$ and $(4,1)$, and of types $(2,3)$ and $(3,2)$, respectively. The explicit map between the parameter $\Delta$ in eq.(3) and $\alpha$ can be readily worked out. For the simulations reported here we have used $\Delta$ 's in the range 0.4-0.6.

The quantum-gravitational proper-time propagator is defined by

$$
G_{k_{0}, k_{4}, \Delta}\left(T^{(3)}(0), T^{(3)}(t)\right)=\sum_{T_{t}} \frac{1}{C_{T_{t}}} e^{-S\left(T_{t}\right)} .
$$

where the summation is over all four-dimensional triangulations $T_{t}$ of topology $S^{3} \times[0,1]$ and $t$ proper-time steps, whose spatial boundary geometries at proper times 0 and $t$ are $T^{(3)}(0)$ and $T^{(3)}(t)$. The order of the automorphism group of the triangulation $T_{t}$ is denoted by $C_{T_{t}}$. The propagator can be related to the quantum Hamiltonian conjugate to $t$, and in turn to the transfer matrix of the (Euclidean) statistical theory [23].

While it may be difficult to find an explicit analytic expression for the full propagator (44) of the four-dimensional theory, Monte Carlo simulations are readily available, employing standard techniques from Euclidean dynamically triangulated quantum gravity 24. Ideally one would like to keep the renormalized cosmological constant $\Lambda$ fixed in the simulation. ${ }^{3}$ The presence of the cosmolog-

\footnotetext{
${ }^{3}$ For the relation between the bare (dimensionless) cosmological constant $k_{4}$ and $\Lambda$ see 11 .
} 


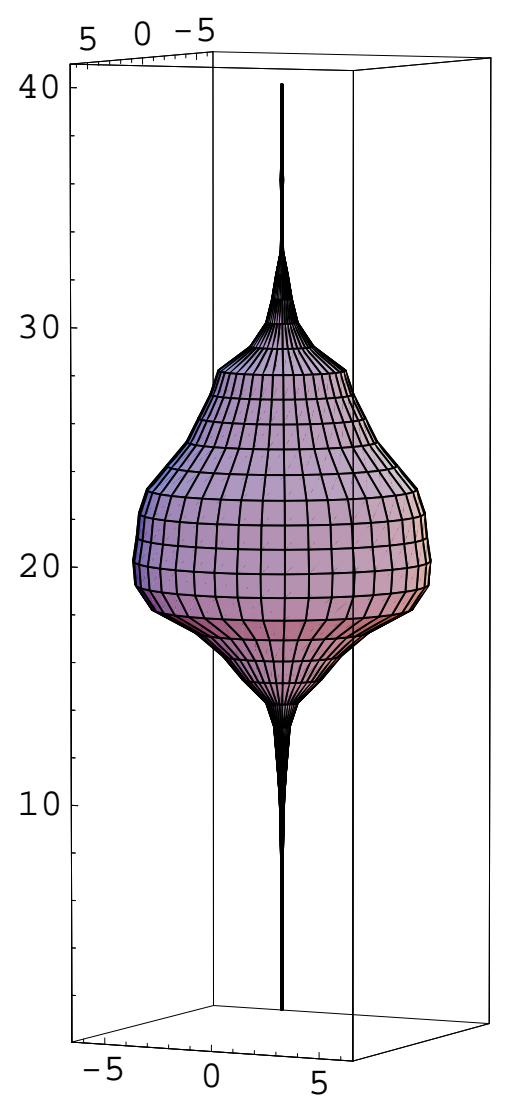

Figure 1: Snapshot of a "typical universe" of volume 91.1k from the Monte Carlo simulations. The total time extent (vertical direction) is $t=40$ as indicated. The circumference at integer- $t$ is the spatial three-volume $V_{3}(t) \times 0.02$ (in units where $a_{s}=$ $1)$. The surface represents an interpolation between adjacent "spatial volumes". No attempt has been made to capture the actual $4 \mathrm{~d}$ connectivity between neighbouring spatial slices.

ical term $\Lambda \int \sqrt{g}$ in the action then implies that the four-volume $V_{4}$ fluctuates around $\left\langle V_{4}\right\rangle \sim \Lambda^{-1}$. However, for simulation-technical reasons one fixes instead the average number $\left\langle N_{4}\right\rangle$ of four-simplices, or $\left\langle V_{4}\right\rangle$, from the outset ${ }^{4}$, effectively working with a cosmological constant $\Lambda \sim\left\langle V_{4}\right\rangle^{-1}$.

Fig 1 shows a snapshot of a configuration with $N_{4} \approx 91100$ four-simplices as it appears in the Monte Carlo simulations. Important information is contained in how the volume $V_{3}$ of spatial slices and the time extent $\tau$ of the observed universe ${ }^{5}$

\footnotetext{
${ }^{4}$ For fixed $\alpha$ (or $\Delta$ ) one has $\left\langle N_{14}\right\rangle \propto\left\langle N_{23}\right\rangle \propto\left\langle N_{4}\right\rangle$. According to 23 we have $V_{4}=$ $a_{s}^{4}\left(N_{14} \sqrt{8 \alpha+3}+N_{23} \sqrt{12 \alpha+7}\right)$. We set $a_{s}=1$.

${ }^{5}$ The time extent $\tau \leq t$ measures the time during which the universe has spatial slices of macroscopic size $V_{3} \gg 1$.
} 
behave in relation to the total spacetime volume $V_{4}$. To good approximation we find that the spatially extended parts of the spacetimes for various four-volumes $V_{4}$ can be mapped onto each other by rescaling the spatial volumes and the proper times according to

$$
V_{3} \rightarrow V_{3} / V_{4}^{3 / 4}, \quad \tau \rightarrow \tau / V_{4}^{1 / 4}
$$

This is illustrated in Fig 2, displaying the so-called volume-volume correlator

$$
\left\langle V_{3}(0) V_{3}(\delta)\right\rangle=\frac{1}{t^{2}} \sum_{j=1}^{t} V_{3}(j) V_{3}(j+\delta)
$$

measured for three different spacetime volumes $V_{4}$, using the rescaling (5), and where we have periodically identified $T_{3}(t)=T_{3}(0)$. The detailed statistical analysis leading to (5), (6) and the measurement of the spatial Hausdorff dimension below will be published elsewhere [25] (see also 21] for an analogous treatment in three dimensions).

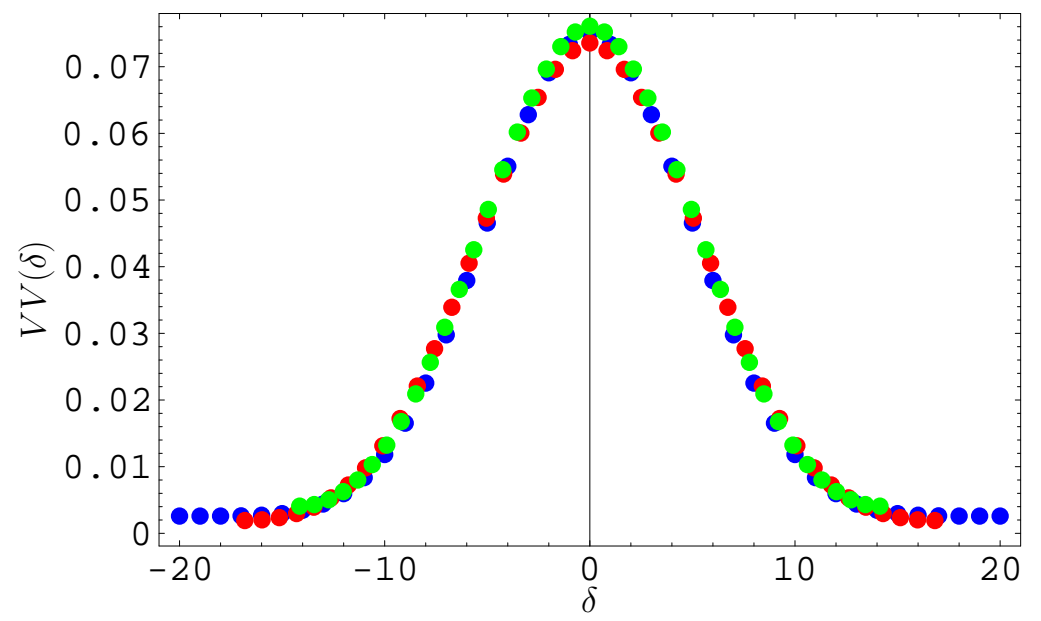

Figure 2: Measurement of spatial volume-volume correlators for spacetimes with $N_{4} \approx$ $45.5,91.1$ and $184.4 \mathrm{k}$, and after performing the rescaling (15).

Relation (5) strongly suggests that what we "observe" in the simulations are genuine four-dimensional universes whose spatial hypersurfaces are threedimensional. To test this we have recorded the average geodesic distance $\langle r\rangle_{V_{3}}$ between points in the spatial three-volumes $V_{3}$. If the spatial geometry is indeed three-dimensional one expects a leading behaviour

$$
\langle r\rangle_{V_{3}} \sim V_{3}^{1 / 3}
$$


Fig 3 shows the results of measuring $\langle r\rangle_{V_{3}}$ in the macroscopic spatial slices of our quantum universes. ${ }^{6}$ It combines the data for universes with total $N_{4} \approx 45.5,91.1$, 184.4 and $371.2 \mathrm{k}$. The straight line in Fig 3 has merely been drawn to guide the

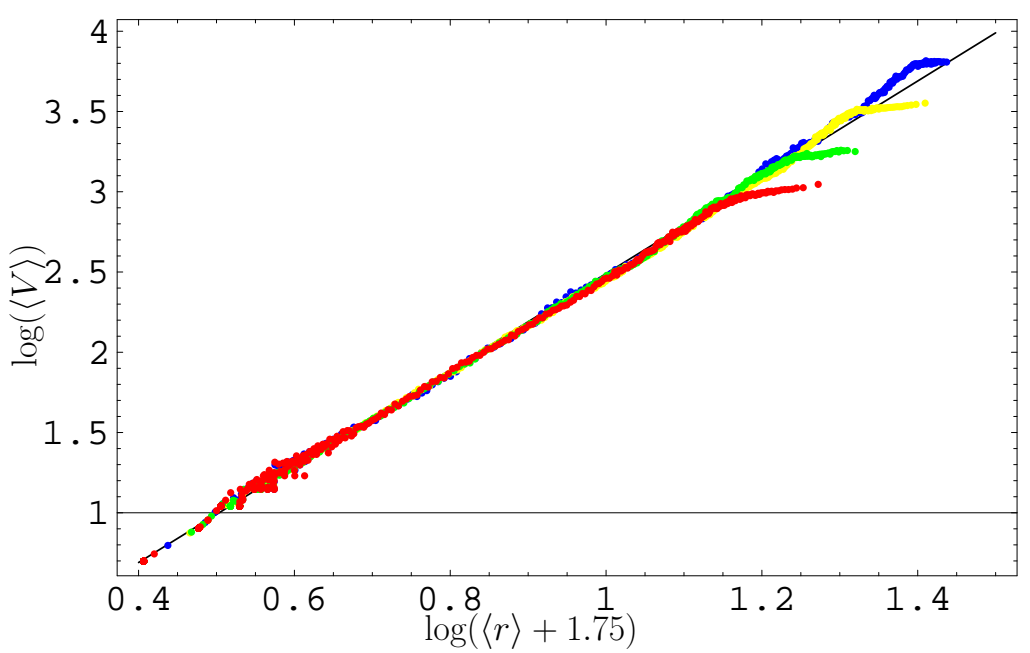

Figure 3: The log-log plot of the average geodesic distance $\langle r\rangle_{V_{3}}$ versus $V_{3}$. It combines data from universes where $N_{4} \approx 45.5,91.1,184.4$ and $371.2 \mathrm{k}$.

eye; it corresponds to the three-dimensional behaviour (17). From these combined measurements, the evidence for three-dimensional slices is rather compelling. A best fit for the spatial Hausdorff dimension $d_{h}$ in the relation $\langle r\rangle_{V_{3}} \sim V_{3}^{1 / d_{h}}$ yields $d_{h}=3.10 \pm 0.15$. As should be clear from the Introduction, reproducing this "classical" dimensionality dynamically from a fully nonperturbative formulation of quantum gravity constitutes a highly nontrivial result. However, it should be kept in mind that a property like $\langle r\rangle_{V_{3}} \sim V_{3}^{1 / 3}$ provides only a crude characterization of the spatial geometry, and by no means implies that the space "observed" in the computer simulations is a nice smooth $3 \mathrm{~d}$ space at short distances. - Further details about the simulations, measurements, fits, and the complete phase diagram of the model will be published shortly [25].

\section{Discussion}

Causal dynamical triangulations are a framework for defining quantum gravity nonperturbatively as the continuum limit of a well-defined regularized sum over geometries. Interestingly, and in complete agreement with current observational

\footnotetext{
${ }^{6}$ The appearance of a small additive constant $\langle r\rangle \rightarrow\langle r\rangle+c, c=1.75$, is a finite-size scaling effect familiar from earlier studies of Euclidean quantum gravity 26, 15] which improves the range of applicability of (7) for small $V_{3}$.
} 
data is the fact that the physical cosmological constant $\Lambda$ in dynamical triangulations is necessarily positive. ${ }^{7}$

Recall that the effective cosmological constant in our simulation is $1 /\left\langle V_{4}\right\rangle$. Since, according to (5), both the spatial three-volumes and the time extension of our quantum universe relate to $\Lambda$ canonically (that is, in a way expected from their classical dimensionality), it is appropriate to call this universe macroscopic.

This leads us to conclude that we have observed the emergence of a fourdimensional macroscopic world with three-dimensional spatial geometries. Judging from the computer simulations, this dynamically generated quantum geometry acts as a background geometry around which small quantum fluctuations take place. Further numerical studies will be needed to make this into a quantitative statement. The situation is really rather remarkable: we started out from an explicitly background-independent formalism and have rederived a particular stable background geometry. Obviously, questions about the nonrenormalizability of perturbative gravity will have to be readdressed in this new context (maybe along the lines outlined in [27]), with the benefit of having an explicit microscopic model of quantum spacetime and its quantum fluctuations. We hope future simulations and analytic studies of the model will teach us how to reconcile these aspects. The final picture may be that of an asymptotically safe theory in the sense of Weinberg [28], or turn out to require a completely new theoretical framework.

\section{Acknowledgment}

All authors acknowledge support by the EU network on "Discrete Random Geometry", grant HPRN-CT-1999-00161. In addition, J.A. and J.J. were supported by "MaPhySto", the Center of Mathematical Physics and Stochastics, financed by the National Danish Research Foundation. J.J. acknowledges support by the Polish Committee for Scientific Research (KBN) grant 2P03B 09622 (2002-2004).

\section{References}

[1] N. Ishibashi, H. Kawai, Y. Kitazawa and A. Tsuchiya: A large-N reduced model as superstring, Nucl. Phys. B498 (1997) 467-491 hep-th/9612115.

[2] H. Kawai, S. Kawamoto, T. Kuroki, T. Matsuo and S. Shinohara: Mean field approximation of IIB matrix model and emergence of four-dimensional space-time, Nucl. Phys. B647 (2002) 153-189 hep-th/0204240.

\footnotetext{
${ }^{7}$ Note that without further observational input the value of $\Lambda$ remains a free parameter of the quantum theory.
} 
[3] H. Kawai, S. Kawamoto, T. Kuroki and S. Shinohara: Improved perturbation theory and four-dimensional space-time in IIB matrix model, Prog. Theor. Phys. 109 (2003) 115-132 hep-th/0211272.

[4] J. Ambjørn, K.N. Anagnostopoulos, W. Bietenholz, T. Hotta and J. Nishimura: Large $N$ dynamics of dimensionally reduced 4-D SU(N) super Yang-Mills theory, JHEP 0007 (2000) 013 hep-th/0003208; Monte Carlo studies of the IIB matrix model at large N, JHEP 0007 (2000) 011 hep-th/0005147.

[5] J. Ambjørn, K.N. Anagnostopoulos, W. Bietenholz, F. Hofheinz and J. Nishimura: On the spontaneous breakdown of Lorentz symmetry in matrix models of superstrings, Phys. Rev. D65 (2002) 086001 hep-th/0104260.

[6] J. Nishimura and F. Sugino: Dynamical generation of four-dimensional space-time in the IIB matrix model, JHEP 0205 (2002) 001 hep-th/0111102.

[7] J. Nishimura: Exactly solvable matrix models for the dynamical generation of space-time in superstring theory, Phys. Rev. D65 (2002) 105012 hep-th/0108070.

[8] J. Nishimura and G. Vernizzi: Spontaneous breakdown of Lorentz invariance in IIB matrix model, JHEP 0004 (2000) 015 hep-th/0003223.

[9] P. Bialas, Z. Burda, B. Petersson and J. Tabaczek: Large $N$ limit of the IKKT matrix model, Nucl. Phys. B592 (2001) 391-407 hep-lat/0007013.

[10] Z. Burda, B. Petersson and J. Tabaczek: Geometry of reduced supersymmetric 4-d Yang-Mills integrals, Nucl. Phys. B602 (2001) 399-409 hep-lat/0012001; Z. Burda, B. Petersson, M. Wattenberg: From 4-d reduced SYM integrals to branched polymers, Acta Phys. Polon. B34 (2003) 4765-4776 hep-th/0308194.

[11] J. Ambjørn and J. Jurkiewicz: Four-dimensional simplicial quantum gravity, Phys. Lett. B278 (1992) 42-50.

[12] M.E. Agishtein and A.A. Migdal: Critical behavior of dynamically triangulated quantum gravity in four-dimensions, Nucl. Phys. B385 (1992) 395-412 hep-lat/9204004.

[13] S. Catterall, J.B. Kogut and R. Renken: Phase structure of fourdimensional simplicial quantum gravity, Phys. Lett. B328 (1994) 277-283 hep-lat/9401026; Singular structure in 4 D simplicial gravity, Phys. Lett. B416 (1998) 274-280 hep-lat/9709007. 
[14] B.V. de Bakker and J. Smit: Curvature and scaling in 4-d dynamical triangulation, Nucl. Phys. B439 (1995) 239-258 hep-lat/9407014.

[15] J. Ambjørn and J. Jurkiewicz: Scaling in four-dimensional quantum gravity, Nucl. Phys. B451 (1995) 643-676 hep-th/9503006.

[16] P. Bialas, Z. Burda, A. Krzywicki and B. Petersson: Focusing on the fixed point of 4-d simplicial gravity, Nucl. Phys. B472 (1996) 293-308 hep-lat/9601024.

[17] B.V. de Bakker: Further evidence that the transition of $4 D$ dynamical triangulation is 1st order, Phys. Lett. B389 (1996) 238-242 hep-lat/9603024.

[18] R. Loll: Discrete approaches to quantum gravity in four dimensions, Liv. Rev. Rel. 13 (1998), http://www.livingreviews.org gr-qc/9805049.

[19] C. Teitelboim: Causality versus gauge invariance in quantum gravity and supergravity, Phys. Rev. Lett. 50 (1983) 705-708; The proper time gauge in quantum theory of gravitation, Phys. Rev. D28 (1983) 297-309.

[20] J. Ambjørn and R. Loll: Non-perturbative Lorentzian quantum gravity, causality and topology change, Nucl. Phys. B 536 (1998) 407-434 hep-th/9805108.

[21] J. Ambjørn, J. Jurkiewicz and R. Loll: Nonperturbative 3-d Lorentzian quantum gravity, Phys. Rev. D64 (2001) 044011 hep-th/0011276.

[22] J. Ambjørn, J. Jurkiewicz and R. Loll: A nonperturbative Lorentzian path integral for gravity, Phys. Rev. Lett. 85 (2000) 924-927 hep-th/0002050|.

[23] J. Ambjørn, J. Jurkiewicz and R. Loll: Dynamically triangulating Lorentzian quantum gravity, Nucl. Phys. B 610 (2001) 347-382 hep-th/0105267.

[24] J. Ambjørn, B. Durhuus and T. Jonsson: Quantum geometry, Cambridge Monographs on Mathematical Physics, Cambridge University Press, Cambridge, UK, 1997.

[25] J. Ambjørn, J. Jurkiewicz, R. Loll and Y. Watabiki, to appear.

[26] J. Ambjørn, J. Jurkiewicz, Y. Watabiki: On the fractal structure of two-dimensional quantum gravity, Nucl. Phys. B 454 (1995) 313-342 hep-lat/9507014.

[27] J. Ambjørn, J. Jurkiewicz and R. Loll: Renormalization of 3d quantum gravity from matrix models, Phys. Lett. B 581 (2004) 255-262 hep-th/0307263. 
[28] O. Lauscher and M. Reuter: Ultraviolet fixed point and generalized flow equation of quantum gravity, Phys. Rev. D65 (2002) 025013 hep-th/0108040; M. Niedermaier: Dimensionally reduced gravity theories are asymptotically safe, Nucl. Phys. B 673 (2003) 131-169 [hep-th/0304117. 